\title{
MARKETING MULTINIVEL VS. ESTAFA PIRAMIDAL: ¿UN NEGOCIO REDONDO?
}

\section{MULTI-LEVEL MARKETING VS. PYRAMID SCAM: A ROUND BUSINESS?}

Gloria María Acosta Alvarez De Hoyle* Anabel Santos Moreno**

* Abogada, MBA, Doctorando en Gestión Estratégica, con diversos estudios de postgrado donde destacan Finanzas internacionales y Marketing global en Harvard University. Auditora participativa de Género por OI|T, Auditora líder en Protección al consumidor Smart Campaign, Auditora líder ISO 9001. Actualmente se desempeña como Coordinadora de Maestrías de Derecho en Escuela de Postgrado Unifé, CEO Saway Perú y Miembro de la mesa de trabajo del Banco Mundial: Educación financiera Perú 2021.

** Alumna noveno ciclo Unifé-Especialidad Derecho Comercial.

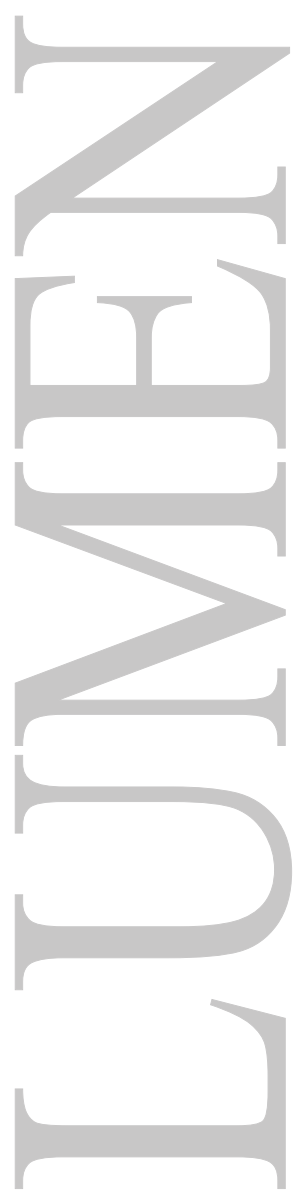




\title{
MARKETING MULTINIVEL VS. ESTAFA PIRAMIDAL: ¿UN NEGOCIO REDONDO?
}

\author{
MULTI-LEVEL MARKETING VS. PYRAMID SCAM: \\ A ROUND BUSINESS?
}

Gloria María Acosta Alvarez De Hoyle Anabel Santos Moreno

\begin{abstract}
Agradecimientos:
A los Representantes de la Superintendencia de Banca, seguros y AFP: Dr. Alfonso Villanueva y Dr. Gustavo Gálvez, por la gentil atención para las investigadoras mediante entrevista respecto de pirámides financieras.
\end{abstract}

\section{RESUMEN:}

Importante precisión de diferencias entre negocio multinivel, correspondiente a marketing de redes y pirámide financiera; ambos potenciados por la velocidad de redes sociales a nivel global, para determinar la existencia de un ilícito penal en cada caso. Se propone una revisión desde el punto de vista del usuario a nivel general respecto de las competencias de los organismos públicos en los cuales podrían - o no - generar algún impacto; nuestro segundo punto de vista es respecto de la propia empresa y la necesidad de responsabilidad, ética y transparencia; y finalmente nuestro tercer punto de vista es desde el regulador peruano.

\section{PALABRAS CLAVE:}

Pirámide financiera, Marketing multinivel, regulación financiera, estafa financiera, Sistema Ponzi, Negocios en red, Marketing de referidos, transparencia, ética, responsabilidad, usuario diligente, consumidor razonable, inversión de riesgo, educación financiera, cultura de negocios.

\begin{abstract}
:
Important precision of differences between multilevel business, corresponding to network marketing and financial pyramid; both boosted by the speed of social networks globally, to determine the existence of a criminal offense in each case. A review is proposed from the point of view of the user at a general level regarding the competencies of public bodies in which they could - or not - generate an impact; our second point of view is regarding the company itself and the need for responsibility, ethics and transparency; and finally our third point of view is from the Peruvian regulator.
\end{abstract}

\section{KEY WORDS:}

Financial Pyramid, Multilevel Marketing, Financial Regulation, Financial Scam, Ponzi System, Network Business, Referral Marketing, Transparency, Ethics, Responsibility, Diligent User, Reasonable Consumer, Risk Investing, Financial Education, Business Culture.

\section{INTRODUCCIÓN}

"El dinero viene del sudor de tu frente" frase célebre de mi padre $\left(G M A A H^{*}\right)$ con una señal tan prominente sobre su rostro en cada día a día ajetreado de trabajo; por lo que NO cierto es que "la plata llega sola" como decía algún expresidente. Resulta claro que un crecimiento sostenible empresarial en cuanto a rentabilidad se refiere es directamente proporcional al riesgo asumido, fruto del trabajo y esfuerzo constante. 
La presente investigación académica parte de una iniciativa de disertación jurídica en las aulas de Derecho de la UNIFÉ, en el curso de Derecho Bancario donde se propuso el caso CLAE como problemática aún actual y vigente; ello puesto que resultaba anecdótico que un conocido locutor radial juvenil había declarado haber sido "estafado" por una pirámide financiera. Y es que si a cualquier ciudadano de a pie le preguntan si quiere dinero fácil, probablemente diga que sí sin siquiera dudarlo, resulta simple naturaleza humana... lo cierto es que no hay mejor método para identificar el riesgo que nos indiquen que el crecimiento exponencial injustificado de la rentabilidad,

El sistema financiero del Perú se ha visto enfrentado, en diversas ocasiones, a las devastadoras consecuencias que pueden tener la informalidad y la opacidad de las instituciones. El abuso y el comportamiento poco ético y delictual de algunos pocos o no tan pocos, ha mellado y debilitado la economía peruana, especialmente a la clase trabajadora de este país, quienes han sido (y pueden ser aún) víctimas de estafas, al no existir; es por ello que presentamos la problemática.

Como consecuencia de lo anterior, actualmente se vienen desarrollando nuevas formas de defraudar, utilizando para este cometido algunas vulnerabilidades de la realidad económica, cultural y social de muchos peruanos, entre ellas se encuentran las pirámides financieras que, a pesar de no ser nuevas estrategias ni absolutamente ajenas han evolucionado en su forma de proceder, con la utilización de medio electrónicos, los cuales facilitan la captación de víctimas y constituyen una nueva modalidad de dar funcionalidad a las pirámides, modalidades cuyos alcances aún no han sido estudiados ni comprendidos en toda su extensión, dado su constante cambio.

Ante el enorme atractivo de una rentabilidad exorbitante y la facilidad de trabajarlo on line los espacios de crecimiento de estas actividades continúan provocando para las potenciales víctimas de este tipo de estafas las promesas de los gestores de la pirámide y su reinvención en el tiempo, con nuevas facetas y formas de operar, la intervención de la Superintendencia de Bancos, Seguros y AFP (En adelante SBS) resulta muy importante, para regular el mercado informal del sistema financiero peruano, con el objeto de tomar las medidas pertinentes para evitar que se siga dañando la confianza de los ahorristas en el sistema..

Desarrollamos a continuación el análisis de los ya conocidos esquemas piramidales y la importancia que tienen para todas las instituciones la confianza del público en el sistema financiero peruano (entendiéndose por este, exclusivamente el sistema formal o regulado), como alternativa para remediar este tipo de fenómenos, siendo la confianza y la transparencia para que una entidad funcione de manera equilibrada y armónica.

\section{Pirámides financieras: Antiguo negocio con nuevas tecnologías de acceso a redes.}

Definimos pirámide financiera como la promesa de obtener enormes utilidades en poco tiempo, presentándose como una solución milagrosa, como aquel camino rápido a la riqueza con el que cualquiera alguna vez ha soñado. Pero la experiencia ha demostrado que este tipo de apuestas es en de alto riesgo y que la mayoría de sus participantes suelen verse profunda e irremediablemente perjudicados, tras el colapso de la pirámide. ¿Qué debemos hacer entonces para identificar este tipo de esquemas fraudulentos?

Información: Lo primero que tenemos que tener en cuenta, es que para realizar una inversión, lo ideal es estar bien informado y tener la seguridad de que el destino del dinero otorgado será utilizado para los fines (inversiones) informadas, así como ser consciente de que las inversiones más seguras son las que tienen tasas de retorno más bajas y que los retornos extraordinarios y fuera de la media establecida en el mercado financiero local, debe ser analizada con detencióni 
Lo segundo que debemos observar es que un sistema solvente por sí mismo, que genera sus propias utilidades, no requiere del ingreso constante, ya sea de capital o de miembros. Por ello, es necesario precisar que la inversión no requiere que los sujetos que aporten dinero al proyecto se recomienden entre sí y que, de ello, quien recomienda reciba una bonificación extra. La inversión per se, es la fachada que utiliza una empresa con naturaleza piramidal. Por lo que, para el público no resulta tarea fácil diferenciar una de otra.

Un ejemplo de pirámide, muy aparte de CLAE, es las "Tejedoras de Sueños" la cual fue denunciada por la periodista Patricia Del Río en el 2016 y difundido por el diario peruano El Comercio. Esta agrupación establecía dos requisitos fundamentales: el aporte de 1.400 dólares y convencer a dos personas más para que contribuyan con sus respectivos aportes. Esta pirámide estaba basada en una temática de empoderamiento femenino que incitaba a las mujeres a ser más solidarias y donar grandes sumas de dineros, las cuales serían recompensadas con grandiosas donaciones, las que en realidad provenían de nuevos miembros de la pirámide. En este tipo de esquema piramidal, la comunicación que se tenía con los promotores, en principio era a través de medios electrónicos, pidiendo que se mantenga en secreto. De esta manera y, siendo investigado por la SBS, se determinó que consistía en una agrupación con "esquema de Ponzi" el 7 de Julio del 2016.

En 1920, Carlos Ponzi, un italiano inmigrante, elaboró una modalidad de estafa basada en una red de captación de "inversionistas" que ofrecía duplicar el dinero a corto plazo, aduciendo que lo recaudado era invertido en la reventa de sellos postales a través de la Security Exchange Company, empresa que constituyó y manejó. No obstante, las ganancias obtenidas por el negocio de compra y venta de estampillas postales no podría ni tendría jamás la utilidad que el señor Carlos Ponzi ofrecía a sus colaboradores e inversionistas; la realidad es que aquellas utilidades pagadas a los primeros colaboradores, prevenían simple y llanamente de los nuevos capitales, provenientes de nuevos inversionistas atraídos por las exorbitantes ganancias prometidas, La SBS determina que las pirámides financieras son "un mecanismo de captación de dinero, con el ofrecimiento de altas ganancias en un corto plazo, sin que exista una actividad económica que lo sustente" (obtenido en línea de SBS INFORMA, Boletín quincenal N011/JULIO 2017). Tal conducta es sancionada como un delito típico, antijurídica y culpable en algunas legislaciones de Europa y Latinoamérica; a este tipo de delito se lo conoce como Estafa Financiera y su elemento diferenciador es, precisamente, que defrauda no sólo a las víctimas resultantes de la piramidación (ahorristas que ven perdidos sus fondos) sino que también, vulneran directamente la confianza de la ciudadanía en las entidades financieras. Por otro lado, en el general de los países, este tipo de infracciones queda bajo el tipo penal general de la estafa y constituye faltas del tipo administrativo y tributario, coligadas de la informalidad en la que suelen trabajar este tipo de estafadores.

Asimismo, siguiendo las palabras usadas por la ASFI "la piramidación constituye un efecto por el que cualquier capital, sea grande o pequeño, se obtiene un elevado rendimiento en corto tiempo sin que se pueda establecer cuál es el destino y fin del dinero invertido y cuál fue la operación financiera que generó la rentabilidad". Los efectos de cada pirámide serán de acuerdo al nivel de participaciones que se obtuvieron durante el periodo de vigencia de la misma junto al número de nuevos participantes, los cuales deben ser exponencialmente mayores a los primeros, para sostener toda la estructura.

Entonces, esta forma fraudulenta funciona, en principio, recaudando fondos con la promesa de brindar intereses altos y a corto plazo, además de pedir a sus "inversionistas" que persuadan a sus familiares o amigos para que ingresen al negocio, muy posible, a cambio de bonos extras o mayores beneficios. 
Como ya se ha insinuado, la razón de que este tipo de fraude se haya relacionado con la forma de una pirámide, es debido a que el primer grupo de aportantes recibirá el pago correspondiente con el aporte del segundo grupo y el segundo con el del tercer grupo, sucediendo esto de manera continua y repetitiva, hasta que las autoridades intervengan o el sistema colapse debido a la falta de sustento, dándose el caso de que, por ejemplo, el número de aportantes en el sexto año se haya reducido considerablemente debido a que no resulta posible seguir devolviendo el dinero a los aportantes de los primeros cinco años y, lógicamente, tampoco a los del sexto año. Sin embargo, hasta ese momento los fundadores y promotores de la pirámide habrán recaudado lo suficiente como para salir del país y realizar algo similar en otro.

\section{Respecto del sistema financiero peruano.}

Esta capacidad predictiva, se grafica en los índices de riesgos y credibilidad que tan ávidamente desarrolla y maneja el sistema financiero en su conjunto, los cuales son una guía para todos los miembros, especialmente para la Banca y otras instituciones financieras. El estudio, la medición, el análisis del mercado y de las conductas humanas son las herramientas desarrolladas por el Sector Financiero, para tomar decisiones consistentes y seguras, que protejan a todo el Sistema Financiero en su conjunto, pero principalmente, para mantenerlos a ellos resguardados.

Por su parte, las personas (es decir los usuarios del sistema financiero), contribuyen a la estabilidad del Sistema Financiero en su conjunto por medio de la confianza entregada. Así lo expresa también el Abogado José María López Jiménez, en su análisis de la normativa y reglamentación española sobre el sistema financiero realizado a comienzos del 2009: "La confianza de los depositantes puede inducir a confusión, pues no queda claro si el cliente debe confiar en todo el Sistema Financiero o sólo en la entidad de crédito del Sistema Financiero a la que confía sus fondos. Nos inclinamos a pensar por que la confianza ha de ser con su entidad, y de la confianza de cada cliente en su particular entidad, llegaremos a la confianza en todo su conjunto del Sistema Financiero." (LÓPEZ, 2009 p.3).

De esta forma y en resumidas cuentas, el Sistema Financiero en general (y en particular en Perú, donde se encuentra compuesto por la Banca, las Aseguradoras y las Administradoras de Fondos de Pensiones, entre otras entidades destinadas a captar ahorros y otorgar créditos, supervisados por la SBS), se sostiene sobre un sistemas de sinergias basados en la confianza y en la credibilidad; las instituciones deben poder creer que los ciudadanos responderán a las obligaciones contraídas con ellos por medio del pago, puntual y completo. De la misma forma, deben confiar en que los índices económicos y macroeconómicos de una economía son veraces, para invertir y emitir los instrumentos financieros que impulsaran el ahorro de los ahorristas, con certeza. En su lugar, lo depositantes y ahorristas deben confiar en que las entidades Bancarias a las que entregan sus ahorros cumplirán su promesa de mantener a resguardo el dinero entregado. Deben confiar además que aquellos acuerdos o instrumentos con un interés o reajuste como retorno, entre otras manifestaciones de confianza.

Pero ¿qué sucede cuando esta confianza no se ha desarrollado aún? ¿O si es quebrantada? ¿Quién es el responsable de desarrollarla? ¿Las entidades financieras? ¿Las autoridades de cada país? Tales preguntas son de extrema relevancia y se han realizado desde múltiples escenarios, incluso desde algunas organizaciones internacionales destinadas a estudiar las causas y soluciones de la pobreza en el mundo, actores que han planteado aún más preguntas, tal como se retrata en la siguiente cita: "Pero quedan aún muchos misterios. ¿Por qué tomó tanto tiempo descubrir que los pobres podían ser sujetos de crédito confiables? ¿Por qué algunos modelos de crédito funcionan bien en algunos países y en otros no? Si bien en todas partes se reconoce el acceso al crédito como una clave para mejorar el bienestar, o sabemos realmente en qué se gastan los préstamos obtenidos." (CONGER, INGA Y WEBB, 2015 p.13) 
La lentitud y el desarrollo poco sostenible en el tiempo que han tenido algunos sectores del Perú, pueden explicarse para muchos en los bajos índices de confianza existentes en el mercado financiero peruano y en la capacidad deficiente de este sector para aproximarse y prestar un servicio necesario y vital para la economía; surtir de liquidez al mercado. También puede explicarse con una aún eminente cultura del ahorro, acompañada de una institucionalidad a momentos opaca y diferencias sociales y culturales muy diversas en un mismo país. No obstante, ninguna de estas explicaciones es suficiente por sí misma. Existen otros actores en deuda.

El primer actor, es la autoridad nacional en materia de control y regulación financiera; la Superintendencia de Bancos, Seguros y Administradora de Fondos de Pensiones. Este organismo ha actuado de forma reactiva en los últimos años y, aunque ha cambiado sus políticas y mejorado en sus mecanismos de control, aún ahora deja aspectos sin controlar, como es el caso de los nuevos desafíos que presentan las redes sociales y los medios de comunicación electrónica.

El segundo actor, son los Servicios Financieros como gremio y como empresas que tienen, en todo este entramado, una responsabilidad social y un rol que jugar. El sector financiero debe plantearse, en su propia estrategia de negocios, la necesidad de trabajar y trazar por una estrategia que convierta a sus instituciones en unas más transparentes y en sus usuarios, en consumidores más informados y empoderados en sus derechos. Solo de esta forma se logrará un sistema de financiero más fortalecido y, por ende, más sustentable en el tiempo.

El último actor debe ser, por cierto, los usuarios o ahorristas. Las personas que interactuamos con el sector financiero debemos asumir la responsabilidad que conllevan nuestros derechos y educarnos sobre el funcionamiento, alcances y límites del sistema financiero. Debemos educarnos además sobre nuestros derechos y ser tenaces al momento de exigirlos; sólo así se logrará equilibrar el balance entre todos estos actores.

Un estudio desarrollado en España, concluyó, después de analizar los perfiles sicológicos y los grados de victimización de diferentes víctimas de los "esquemas Ponzi" (u estafa piramidal) concluyó que: "no existen diferencias entre los factores que favorecen que los entrevistados sigan invirtiendo en la empresa piramidal, estando estos relacionados con la creencia previa de que el negocio es seguro." (PAREJO-PIZARRO, 2017 p.66). En otras palabras, lo anterior se traduce que no existen diferencias sustantivas entre las diferentes víctimas; va más allá de la clase social, educación, grado de formación, género, nacionalidad, etc. La verdadera diferencia, pareciera encontrarse en la aversión personal al riesgo que puede tener cada víctima al riesgo, la cual solo se desarrolla con una consciencia de las consecuencias a largo plazo de apostar por la informalidad o de, simplemente, ignorar las señales que se revelen en el camino.

Un sistema financiero fortalecido es aquel en que todos sus actores entienden su función e interactúan en un mercado con transparencia y fluidez, comprometidos con decisiones que se ajusten a la legalidad, pero también, a los principios y exigencias de un modelo y sistema por sí mismo solvente y sustentable, para todas y todos.

\section{Pirámide Financiera:}

Marketing Multinivel

Análisis:

a. Desde el punto de vista del usuario

b. Desde el punto de vista de las empresa

c. Desde el punto de vista del regulador 


\section{Conclusiones}

¿Qué es una pirámide financiera?

¿Qué hace la SBS? Supervisa y regula, pero no solo eso, sino que dentro de su función está el fomento de la inclusión financiera. ¿Qué es eso? Que todas las personas tengan el acceso y uso de los productos (y servicios) financieros, pero de manera informada. Para que ello suceda tiene que haber educación financiera.

La SBS tiene 4 órganos de línea y muchos otros de apoyo. Los órganos de línea son la Superintendencia adjunta de Banca, Seguro, UIF y siendo el cuarto, AFP. Todas esas cuatro, reportan al superintendente de Banca. Hoy la superintendenta de Banca, Seguros y AFP, es Socorro Heysen Zegarra.

Un área adjunta de Banca perteneciente a la Superintendencia es aquella que supervisa lo relativo a la informalidad empresarial.

Toda empresa que es informal, no está regulada. La función principal de la SBS y AFP, es proteger los depósitos de ahorro del público. Que las personas duerman tranquilas sabiendo que has depositado tu dinero en una entidad patrimonialmente sólida, segura, que no va a quebrar, porque está supervisada. Y la supervisión significa eso, regular todo un esquema estratégico para que cada entidad financiera esté protegida, tenga un patrimonio sólido.

Los bancos reciben depósitos y con estos depósitos, los bancos prestan. Los depósitos son secretos, están protegidos por el secreto bancario, entonces, ¿Cómo tú, como SBS, supervisas que los bancos den adecuadamente o cuiden que están personas tengan confianza? A través del crédito, la SBS controla y revisa cada crédito que las entidades financieras otorgan, la SBS controla y revisa cada crédito que las entidades otorgan. Porque si los créditos están bien otorgados, funciona la cadena de pagos.

"soy cliente, vengo y deposito, banco: con esa plata presto, recupero, y pago al depositante" y no solo tengo que pensar bien en que tengo que pagar, sino que tengo que calzar bien los plazos que yo como entidad financiera estoy prestando. Si viene una persona a depositar un depósito a plazo y dice, yo le voy a pagar como banco en un año. Yo no le puedo prestar a esa misma plata a otra persona en 2 años, porque cuando venga el año, no le voy a tener para pagar.

¿Qué cuida la SBS? Cuida el dinero de los depositantes. Entonces, lo que se ve y se cuida es desde atrás, desde la captación. Para nosotros, una empresa informal es: aquella que capta, que pida deposita aquí y, yo te pago un interés. Ahí, como empresa informal, estoy captando, estoy recibiendo.

- Tú puedes prestar libremente.

Con tu plata, eso no controla, porque eres una empresa prestamista. Lo que se sanciona es que se reciba dinero de la gente y, se brinde un interés. Lo que la SBS hace es: autorizar el funcionamiento de las entidades financieras y las entidades que van a funcionar en el sistema financiero. Por ejemplo: autorización para los bancos, financieras, cajas rurales, municipales.

Si se quiere realizar una de esas actividades, es requerible se presente una solicitud a la SBS y esta, va a requerir requisitos mínimos de capital mínimo que tienes que tener dependiendo del tipo de empresa.

- Se sanciona la captación. 
Ellos tienen que controlar en un área que tienen ahí, van y observan en las provincias que se están dando. Paseas por ahí y ves que dice: "Inversiones XL", y te preguntas si ha sido aprobada por la SBS. ¿O reciben un depósito, ha sido aprobado? No. Se va con un equipo, 3 a 4 personas, se realiza una supervisión inopinada. Está captando, usted no puede y "pam", se cierra la empresa. Se sanciona.

Las empresas informales como las pirámides son controladas de esa manera, pueden llegar una referencia de un afectado o de alguien que tenga conocimiento. Una empresa que está dando un interés por el depósito que se le está brindando, por si acaso. Entonces yo llevo un equipo y veo. O me voy a provincia y veo, que empresas se están captando el dinero de la gente. Porque la captación de fondos es muy delicada, recibir una tasa muy superior del valor del mercado, causando problemas para el consumidor financiero. Para esa persona que cree que esa va a pagar el interés.

\section{4.-Sostenibilidad de la Pirámide}

¿Cómo se sostiene? La pirámide se alimenta de la última persona que va depositando. Los primeros van a recibir los depósitos que se van captando, el ejemplo más claro fue CLAE. CLAE nunca dejó de pagar, iba a reventar de todas maneras y, no estaba supervisada por la SBS a operar. Es por eso que se interviene.

4.1. La estructura que posee una empresa multinivel para el desarrollo de su actividad comercial dista un paralelismo con el de un esquema Ponzi, por lo que el determinar una diferencia resulta muy difícil para el ojo del consumidor, pues existe una compraventa de productos. Al respecto, Peter Vande Nat, Economista Senior de la Comisión Federal de Comercio de EE.UU. deja entrever una diferencia importante señalando que "Una organización es un esquema piramidal cuando los participantes obtienen más beneficios monetarios de reclutar que de vender productos y servicios a los consumidores". Siendo esto así, ¿cuáles son los criterios que adopta actualmente para bases a tomar en cuenta para diferenciar ambas figuras?

La empresa multinivel, no conozco como funciona, pero no aplica acá. Es privado. No recibe dinero del público. Trabaja con productos. Hace una red de mercadeo, vende productos. Para la superintendencia, ello no es de importancia, es legalizado.

Cuando se dirige a una persona de la superintendencia, un regulador como yo. Lo que se sanciona es la captación. Recibir dinero de las personas para prestarlo. Las empresas que prestan dinero por internet, no están supervisadas por la SBS, porque prestan dinero, mas no captan dinero. Con tu patrimonio puedes hacer lo que quieras.

El principal problema de la informalidad es la captación, no se puede captar si no está autorizado por la SBS. Lo mismo que se hace ahora con la cooperativa, a partir de este año. ¿qué hace la SBS? Dice, inscríbanse hasta tal fecha, en el registro de cooperativas. Solo va a reconocer a las cooperativas inscritas y, si no lo haces y captas dinero de los socios, te cierro. Eres informal.

Lo multinivel es un aspecto que no ve la SBS. Es otro tema. Lo importante aquí es la informalidad. Multinivel es un negocio de red de mercadeo, fuente de mercadeo muy rica.

4.2 ¿Es la denuncia de los consumidores la principal fuente de información para alerta de la SBS o existen otros canales por los que se inician procesos de investigación? Reconocemos los principales en la web a través de un correo electrónico que puede ser anónimo inclusive.

Sí, normalmente ubicamos dinero informal porque "les tiran dedo", porque los intereses son muy grandes o están cobrando tasas altas. Normalmente denuncian esta actividad. Puede ser un vecino 
que diga: "me están pidiendo dinero y, me van a pagar una tasa alta de intereses que es de $10 \%$ o $15 \%$ mensual".

Siempre que veas tasas exorbitantes que te paguen ahorro, hay algo raro ahí. Si alguien te dice que le des 500 y te ofrece que en una semana te va a dar 540 o 600, es mucho; puede cumplir, pero en la siguiente semana tal vez no. Eso es informal y sancionable, porque no se puede captar sin la autorización de la SBS.

Sí, un vecino puede denunciar; pero también contamos con un área de informalidad que tenemos una relación en la cual se ve supuestos de algunos potenciales riesgos en este tipo de delitos. Personas, casas, lugares, en las cuales se realizan visitas inopinadas.

Por ejemplo, se llega a una casa y resulta que no hay captación, solo préstamos y, eso no se puede sancionar.

Sí, pueden ser vecinos, la comunidad misma quienes realicen las denuncias. Pero aparte, la Superintendencia Nacional de Bancas y Seguros y AFP tiene un área específica que hace visitas inopinadas a supuestas personas que están captando recursos de terceros sin supervisión de la SBS.

Esa área se llama departamento de asuntos contenciosos a cargo de Carlos Cueva, este departamento depende de la superintendencia adjunta de jurídica.

Dr. Gálvez, estuvo trabajando hasta el año 2013 en informalidad.

Lo que hacía en mi tiempo era recibir denunciar y en base a eso hacia visitas de incognito para verificar si se estaba realizando el acto informal. Lo que se hacía básicamente era buscar denuncias y buscar posibilidades de estas inconductas. Lo primero es verificar la ocurrencia del hecho y, recabar pruebas, como, por ejemplo: hacer depósitos si era una entidad informal que está captando depósitos. O contratar la cobertura de un seguro, por ejemplo, en el caso de las AFOCAT ${ }^{1}$ informales, que son pequeñas aseguradoras de vehículos de transporte público a efectos de accidentes de tránsito. Actualmente hay 46 AFOCAT supervisados por la SBS y sigue habiendo muchos que son informales.

Básicamente, lo que se sanciona son dos aspectos, la captación de fondos y la ejecución de cualquier actividad supervisada por la SBS sin la autorización de la SBS. Y en ese supuesto cae el tema de las AFOCAT, porque están realizando una actividad que está bajo la supervisión de la SBS que es la cobertura de estos siniestros de transporte público sin autorización de la SBS.

\section{5. ¿Qué diferencia hay entre la empresa multinivel y las empresas de estafa piramidal?}

Lo que ocurre en las redes multinivel, lo que están pagando a la persona es el resultado de parte de los ingresos de la empresa, hablando de comisiones. Hay toda una estructura.

Ahora, que haya empresas multinivel que en realidad sean pirámides y que sean un disfraz, es otro asunto. El mismo Herbalife, está acusado de eso. Esa podría ser una de las más grandes pirámides del mundo y nadie sabe. Y en noticias, hay acusaciones a Herbalife.

La Asociación de Fondos Regionales o Provinciales contra Accidentes de Tránsito (AFOCAT) nació como una alternativa económica a las compañías de seguro en la emisión del Seguro Obligatorio contra Accidentes de Tránsito (SOAT) para las unidades de transporte público. Se tratan de organizaciones sin fines de lucro que, compuestas por transportistas de una zona específica (provincia o región), aportan a un fondo que se encarga de cubrir los gastos asociados a un accidente de tránsito de alguno de sus miembros. 
Un caso que, en mi opinión, sí era una pirámide que operó en el año 2008-2009, es "Travel One". "Travel One" era una empresa que te vendía paquetes de promociones turísticas, de cupones para utilizarlas en cruceros con descuentos. Y, ¿qué pasaba? Una vez que comprabas eso, si captabas más gente, te ganabas más plata. Te ganabas una comisión. Pero en estas cosas casi nadie las usaba al final, estas cuponeras nadie las usaba.

Entonces lo que hacía la gente era entrar a "Travel One" para captar gente y recibir más dinero.

- En el caso de Herbalife, ¿Cómo se prueba la captación?

DR. Villanueva: Ese tendría que ser un caso a nivel mundial. Aquí, es una cosa que ni se ha pensado en hacer. En lo que deberías concentrarte es la captación ilegal, aquella que no esté regulada por la SBS o, cualquier operación que la SBS no hay autorizada por la Ley de Banca.

DR. Batrick: En el caso de multinivel, una que realmente lo es, como Avon o L'ebel, se está vendiendo cosméticos y se recibe comisiones. En el caso de Herbalife que vende productos en el que supuestamente te baja de peso y no funciona, se dice que está disfrazando una pirámide, es algo que no podemos afirmar.

Travel One, el caso que te demostré en el que no estás vendiendo nada, solo se enfoca en la captación de nuevas personas, ese viene hacer esquema.

Pero la SBS jamás se ha metido en eso, en una pirámide disfrazada de multinivel, es difícil de probar.

3. Al tratarse de múltiples personas naturales quienes administran redes digitales de aportantes y potenciales aportantes, inclusive bancarizando dichos aportes. ¿La SBS trabaja de forma preliminar con el Ministerio Público para identificar estas prácticas o entrega la información directamente para realizar la investigación en fiscalía?

En nuestra época, las investigaciones se hacían paralelamente. Nosotros hacíamos una investigación, sacábamos un expediente y nos preparábamos para la intervención. Éramos como 10 personas, gente de diversos departamentos, de contenciosos, de seguridad, de logística, de comunicaciones. Era todo un equipo para la intervención.

Por ejemplo, si era en provincias teníamos que viajar y estar todo el día en el hotel, esperando que primero pase denuncia a fiscalía. Y una vez que el fiscal haga suya la denuncia, conseguir la autorización del juez para la orden de clausura.

Teníamos que ir con la carpeta a la fiscalía y que el fiscal haga una denuncia y, pida ese día o al día siguiente, una orden al juez para el cerraje, una orden de intervención.

Una vez ejecutada la clausura de investigación y denuncia ante el poder judicial, ya quedaba en manos de fiscalía. Nuestra función era en el comienzo, en la clausura y, luego éramos parte en el proceso, pero el proceso ya lo llevaba fiscalía.

Frente a una situación en la que las personas se encuentran en un esquema Ponzi, la pregunta principal que se plantean es: “¿a quién debo recurrir?"

La relación entre el potencial aportante y la entidad piramidal podría recaer en lo difuso, pues ¿qué clase de relación tienen? Es potencialmente una relación entre privados, entonces, ¿debería regirse por las normas del Código Civil? Existe asimetría de información y el desenvolvimiento de su 
relación es equiparable a la de un banco, entonces se trata de una relación proveedor-consumidor, ¿Bajo qué ley es sensato ampararse y bajo qué autoridad? A) La Ley General del Sistema Financiero y del Sistema de Seguros y Orgánica de la Superintendencia de Banca y Seguros y acudir a la SBS. B) Ley de protección y defensa del consumidor y acudir al INDECOPI.

En el caso de haberme percatado muy tarde y habiéndose ya concretado la inversión, entonces, ¿la Autoridad encarga debería ser la Superintendencia de Mercado de Valores?

\section{a) INDECOPI}

Si bien es cierto, la relación aparente entre en los involucrados en un esquema Ponzi puede ser similar a una relación proveedor-consumidor, hay que tener en cuenta algunos aspectos. En la Ley № 29571 referente al Código de Protección y Defensa del Consumidor, se establece la defensa del consumidor frente a una situación de desventaja debido a la asimetría informativa.

Sin embargo, al tratarse este de un servicio financiero, el artículo 81 del mismo código ampara en su marco legal la protección del consumidor frente a aquellos servicios financieros prestados por "empresas supervisadas por la Superintendencia de Banca, Seguros y Administradoras Privadas de Fondos de Pensiones", mas no hace referencia a las empresas que no son supervisadas. Teniendo claramente con esto un vacío en la norma con respecto al tema, dado que en estricta aplicación del principio de legalidad, la norma debería hacer mención de las entidades actualmente supervisadas y aquellas que no estándolo, debiesen estarlo bajo las normas y principios de la propia Ley sectorial.

Asimismo, reconoce la regulación y supervisión del sistema financiero y los productos y servicios financieros en poder de la SBS, haciendo hincapié en el principio de especialidad normativa.

\section{b) Superintendencia de Mercado de Valores (SMV)}

La SMV es la entidad encargada de supervisar y dar cumplimiento a las normas que regulan el mercado de valores, la promoción y la incursión en la bolsa de valores e todo tipo de entidades. Por ello, es imposible no pensar en ella cuando lo que se tiene presente es que se ha realizado una inversión que requiere un retorno y en lo posible una ganancia.

Dentro de las funciones de la entidad se encuentran:

- Las personas naturales o jurídicas sujetas a la supervisión de la Superintendencia de Banca, Seguros y Administradoras Privadas de Fondos de Pensiones (SBS) lo están también a la $\mathrm{SMV}$ en los aspectos que signifiquen una participación en el mercado de valores bajo la supervisión de esta última.

- Hay que tener presente el supuesto de que la entidad piramidal, en su mayoría, no realiza movimientos en la bolsa de valores, justamente para evitar ser supervisada por la SBS y la SMV. Sin embargo, en el supuesto en que, si lo hiciera, hay un factor importante que se aplica en este caso al igual que en el de la INDECOPI y corresponde a la aplicación del principio de especialidad normativa, la cual le da plena operatividad a la Superintendencia de Banca, Seguros y AFP.

\section{c) Superintendencia de Banca, Seguros y AFP. (SBS)}

Las entidades financieras son reguladas por la Superintendencia de Banca, Seguros y AFP de autonomía reconocida constitucionalmente, siendo que este órgano se encuentra en las facultades 
de controlar las empresas que administran el ahorro del público, con el fin de salvaguardarlo y mantener el equilibrio en la economía peruana.

Una de las funciones que cumple la SBS es la de brindar seguridad de que las entidades están siendo reguladas de la manera correcta y, por ende, confianza en la entidad financiera regulada.

Por ello, si existiere alguna empresa que use un esquema parecido a la piramidal pero que conste de sustento y cumpla con los requisitos legales para su funcionamiento, este se encontrará registrado por la SBS y será transparente en cuanto a la información que ofrezca.

Ante este contexto, la función sancionadora de la SBS en caso de pirámides financieras fraudulentas se encuentra sustentada en el numeral 1 del artículo $11^{\circ}$ la Ley 26702 Ley General del Sistema Financiero y Sistema de Seguros, siendo que señala que las entidades que carezcan de autorización por parte del a SBS, están prohibidas de administrar fondos del público tal como las entidades financieras reguladas y, sobre todo a "...captar o recibir en forma habitual dinero de terceros, en depósitos, mutuo o cualquier otra forma, y colocar habitualmente tales recursos en forma de ... inversión..." . Incluso en el mismo artículo expresa que se entenderá que una empresa incurre en estas infracciones cuando "se invite al público a entregar dinero bajo cualquier título...".

\section{Regulación de las pirámides}

La forma como se ha regulado en la normativa peruana la competencia de la Superintendencia de Banca, Seguros y AFP le entrega la suficiente amplitud para pronunciarse sobre todo tipo de actividades que, aún actuando de formas diversas y fuera de la legalidad, capten algún tipo de capital. Ello señala un supuesto bastante claro en el que calza el actuar de una pirámide financiera fraudulenta y, ante ello, la sanción correspondiente por la captación de dinero encuentra sustento en el artículo $351^{\circ}$ de la misma ley, la cual señala que la Superintendencia está autorizada de cerrar locales en los que realicen operaciones no autorizadas, con la intervención de un fiscal de la Nación. Siendo que está autorizada a incautar documentación que encuentre y, si es necesario, con el apoyo de la fuerza pública, sin que la SBS incurra en responsabilidad.

Posterior al cierre del local y la incautación de documentación, la SBS formulará denuncia que corresponda al actuar, en este caso defraudación y apropiación ilícita.

Asimismo, en el artículo $352^{\circ}$ de la mencionada ley, se expresa que la SBS está facultada de pedir ante la Corte Suprema la disolución de la entidad infractora.

\subsection{Marco Penal}

De acuerdo a nuestro marco penal, en el art. $246^{\circ}$ del Código Penal se reglamenta a las instituciones financieras ilegales en tanto se produzca la captación habitual de recursos del público en cualquier modalidad, los agentes responsables podrían adquirir en sentencia una pena privativa de libertad de entre tres a seis años y, que se agravaría por el uso de medios de comunicación en un periodo de entre cuatro a ocho años de cárcel, ello sin contar con los díasmulta correspondientes.

Para esto, es suficiente determinar que son instituciones ilegales las que no cuentan con permiso por parte de la Superintendencia de Banca, Seguros y AFP, como ente regulador en caso de captación del ahorro público y la cual contribuirá en la investigación antes de formular denuncia a través de la Unidad de Inteligencia Financiera (UIF). 
Por otra parte, la agravante del uso de medios de comunicación como las redes sociales, podría convertirse en una constante en los casos de pirámides financieras, valga para ello el ejemplo de "Telar de mujeres", caso para el cual se utilizaba tanto las Redes Sociales (como Facebook) como otros sistemas populares de mensajería electrónica como Whatssap. Para tal sentido, la publicación abierta y desmedida de un medio fácil de ganar dinero a través de aportes dinerarios con rentabilidad envidiable, no parece del todo efectivo si no se interpone la confianza entre las personas a las que se va a realizar la invitación, puesto que consta de un convencimiento personalizado y no de una oferta pública y directa.

7. Formas y estrategias de difusión, ocultamiento y crecimiento de estafas piramidales. Análisis a través de casos.

\subsection{CLAE}

El Centro Latinoamericano de Asesoramiento Empresarial, también conocido por sus siglas CLAE fue la empresa bajo la cual se constituyó la más grande estafa piramidal en la historia del Perú.

Fue fundada en 1978, con un capital del valor de 100 mil soles, por Carlos Manrique Carreño, quien en un inicio ofrecía servicios de asesoramiento y quien posteriormente fue convirtiendo a CLAE en una empresa que buscaba captar dinero y devolver un monto superior a la inicial y, como típica entidad basada en un sistema piramidal, ofrecía bonos extra a quienes recomendaban a CLAE a más personas y los hacían participes del negocio de las inversiones. El prometía entre los ahorristas que entre más miembros, mayor "rentabilidad".

Esta empresa duró aproximadamente 16 años, lo que influyó en la confianza de las personas, pues el escuchar el nombre CLAE en los años 90, significaba el sinónimo de rentabilidad en inversiones, dinero fácil, pero confiabilidad.

El tiempo de vigencia, los intereses exorbitantes y los testimonios de las personas que sí percibían altos retornos, significó más para el ahorrista que la seguridad que podría brindarle una entidad regulada; pues CLAE ofrecía lo que estas no, un futuro asegurado con el dinero que irían a percibir.

Por ello, CLAE llegó a manejar US\$ 640 millones sin rendir cuentas a ninguna entidad reguladora y con ello, estafó a cerca de 200 mil personas. Incluso por la cantidad de dinero manejado, se dice que llegó a configurarse el manejo del " $40 \%$ de la liquidez del sistema financiero peruano."

Tanta fue la atención que atraía CLAE, que se decía que, en su periodo de auge (1989-1992), suscribía miles de contratos de depósitos a su nombre. Sin embargo, no solo se trataba de simple atención, el contexto histórico-económico de aquel entonces era demasiado preocupante para la población en general, pues se suscitaba el primer gobierno de Allan García, los rezagos del proyecto de estatización de la banca y el inicio del gobierno de Alberto Fujimori fueron fundamentales para influir en la opinión del público a sentirse proclives por "alternativas" a la Banca formal.

El quiebre de la pirámide CLAE se inició por una disminución en el número de nuevos aportantes, lo que originó que no hubiera fondos suficientes para costear los altos intereses que se ofrecían.

CLAE había colapsado en 1992, burlando a personas pobres y ex-trabajadores que perdieron sus empleos durante la recesión de 1991 y 1992. Los fondos habían sido absorbidos por CLAE, que pagaba tasas de interés de hasta $20 \%$ cuando los bancos convencionales pagaban alrededor del $6 \%$.

En 1992, durante el gobierno de Fujimori, la SBS decidió regular la empresa, pero, Manrique no logró sustentar el estado financiero de la entidad, tampoco logró cumplir con los requisitos que 
le exigía la SBS. Lo cual resultó en que el golpe final fuera la orden de disolución de la empresa el 16 de mayo de 1994 y, como era de esperarse, la denuncia contra Manrique por defraudación y apropiación ilícita.

\subsection{Caso "Telar de mujeres"}

"Telar de mujeres", "Telar de sueños" o "Flor de la abundancia" son nombres que se le daba a una organización que tenía como fin el estafar mujeres bajo la modalidad piramidal, desarrollándose en países como Colombia, España y Argentina, aun siendo que en esta última también participaban hombres.

El fin es sencillo: "cumplir tus sueños en base de solidaridad femenina", pues lo que ofrecen es un 'regalo con mucho amor', siendo que no solo deben guardar confidencialidad acerca de las actividades realizadas, sino que también deben buscar que otras 2 mujeres de confianza se sumen a la causa. En reuniones o a través de las redes sociales una mujer habla con otras (familiares o amigas) y las convence de invertir -debe lograr que al menos dos lo hagan, cada una con un aporte de US\$ 1,200-. La primera recibe la ganancia por "reclutar" a las otras, y estas también por captar a nuevas "inversionistas".

Al igual que en un esquema piramidal, lo que ofrecía esta organización era una rentabilidad por encima de los estándares del sistema financiero formal, llegando a ser 8 veces más del monto invertido, siento este el de 1.400 dólares americanos. Claro que, el monto de inversión podía variar de acuerdo a la forma del "juego".

Asimismo, el incorporar a otras 2 personas como requisito (también constituye una característica importante del esquema Ponzi), el adecuar la estabilidad de la organización en corriente a lo aportado por los 'nuevos'. De lo que se evidencia no existe un respaldo económico solvente.

El uso de medios electrónicos como Facebook y Whatsapp, no solo evidencia que nos encontramos en el auge del siglo XXI y la globalización, sino importa una tarea más difícil en las investigaciones que se puedan realizar a dichas grupos que solo dependen de estas formas de comunicación, obviando por completo la existencia de un domicilio comercial. No obstante, involucra una agravante al delito de intermediación ilegal financiera, como se verá más adelante.

Además, bajo este ejemplo, podemos hablar que en muchos casos, las entidades, grupos $u$ organizaciones que realizan esta actividad ilegal aprovechan de la confianza que tienen los aportantes, o futuros aportantes, no a la entidad que organiza, sino a aquellos que realizan el llamado, los promotores que en un inicio fueron los primeros aportantes y que pueden ser familiares o amigos más íntimos. Pues, "solo se invita a mujeres en las que se "confía y aprecia"."

En este caso, lo que buscaban era que esas dos mujeres llamadas a sumarse a la causa no solo tengan el interés de formar parte de ella, sino que también sean de confianza. ¿Por qué? Pues, porque de esa forma es más fácil que puedan convencerlas de seguir invirtiendo y sigan recomendando personas de confianza. Además de mantener la fachada de una hermandad.

\subsection{Caso Bernard L. Madoff}

Este caso es uno de los más conocidos en EE.UU. pues pasó desapercibido por muchos años (20 años) y causó conmoción en la más alta esfera económica-social. Pues, "borró US\$17,500 millones en capital y más de US\$ 40,000 millones en ganancias falsas" 
Madoff creó su propia correduría bursátil en 1960 denominada Madoff Investment Securities, siendo que, teniéndola de 'fachada' realizó estafas por sumas importantes a bancos, aseguradoras, fondos de inversión, entre otras; usando el esquema Ponzi.

Sus movimientos consistían en ofrecer una rentabilidad de $10 \%$ y $12 \%$ anuales, lo cual no era muy exagerado para la bolsa. Luego, solo invertía una pequeña parte del monto y lo demás lo usaba para pagar a los primeros clientes.

Lo peculiar, es que, en el caso de Madoff, él no pedía a los clientes sus recomendaciones, sino que él aprovechaba de su reconocimiento social para conseguir clientes que, estaban más que dispuestos a aceptar la invitación que este ofrecía. Madoff aprovecha su posición como presidente de NASDAC para que los clientes, continúen invirtiendo y no retiren sus ganancias, asegurando "estabilidad" a su pirámide.

No obstante, todo se vino abajo cuando se dio la crisis económica en EE. UU en el 2008, pues ante el desequilibrio financiero, los inversionistas requerían retirar su dinero para estabilizar sus empresas. Dinero que no podía devolver porque este en realidad solo circulaba entre los antiguos inversionistas y el mismo Madoff. Tres meses después de ser arrestado, se declara culpable y posteriormente es condenado a 150 años de prisión, además de tener que devolver el dinero que utilizó en sus estafas.

En el 2017, se realizó la primera ronda de entrega de cheques para las víctimas de Madoff, siendo un fondo de US\$ 4,000 millones del Departamento de Justicia de Estados Unidos, siendo que pasaron más de 8 años desde su arresto. Y aun cuando se han aceptado más de 35,000 solicitudes por un total de más de US\$ 6,500 millones de pérdidas.

Asimismo, interesante resulta el hecho que, al ser una correduría bursátil, esta estaba siendo supervisada por el SEC (U.S. Securities And Exchange Commission), el regulador del mercado, que no logró descubrir a Madoff hasta que sucedió la crisis. La cual aún sigue siendo una duda.

Las diferencias con el caso CLAE y Tejidos de mujeres, son muchas, teniendo en cuenta que las personas a las que estafó eran personas cultas en el ámbito de los movimientos y las inversiones en la bolsa, sabiendo los riesgos que esta ocasiona. Sin embargo, no deja de llevar la estructura Ponzi, interrelación de un dinero no circulable.

\subsection{Caso Herbalife (Esquema Multinivel)}

Mark Hughes fundó en 1980 la empresa multinivel más cuestionada en la última década acusada de ser un esquema piramidal, se trata de Herbalife.

En el 2012, Bill Ackman, director ejecutivo de Pershing Square Capital, declaró en una conferencia que la compañía Herbalife está destinada a fracasar, acusando de ser un esquema piramidal y, en tanto sus acciones bajen a cero, él ganaría cerca de mil millones de dólares. Seguida a esta entrevista, realizó constantes declaraciones en los medios de comunicación.

Ackman declaró realizar una investigación a profundidad, en la que encontró un desequilibrio en los ingresos que percibe la empresa y en su política. Aseguró que los ingresos de Herbalife se sostienen de acuerdo a la cantidad de reclutamientos que realicen los distribuidores, creando así un sistema en líneas ascendiente y descendiente de transferencia de dinero por las comisiones obtenidas de las ventas de aquellos que recién ingresan al negocio. Y que los precios elevados de estos productos solo contribuyen a la fachada. 
Así pues, aseguró a la prensa que los clubes de nutrición creados por los supervisores de Herbalife, presentarían una pérdida de 12 millones de dólares si sólo se enfocara en vender los productos. Esta declaración, deja entrever que aquellos clubes solo perduran si se utiliza para reclutar más distribuidores ganando una comisión por sus ventas.

En el documental 'Betting on Zero' dirigido por Ted Braun, se reconoce la existencia de un grupo activista de personas en contra de Herbalife por causarles perjuicios en tanto no se cumplió con el objetivo, adquirir ingresos que superen los gastos realizados en la compra de productos, tachándolo de ser una empresa multinivel con esquema piramidal.

En el año 2013, Carl Icahn tomo el $26.2 \%$ en acciones de Herbalife, haciendo que de alguna forma la empresa lograra estabilizarse.

El 15 de julio del 2016, la Comisión Federal de Comercio de los Estados Unidos acusó a Herbalife con cargos de prácticas, injustas, falsas y fraudulentas. Y la multa a Herbalife fue de 200 millones de dólares, después de realizada una investigación. Sin embargo, la Comisión nunca denominó a Herbalife como una empresa con esquema piramidal; siendo de ayuda para que Michael Johnson, hasta entonces director ejecutivo de la empresa, determinara un triunfo y siguiera adelante con la compañía, perpetuando el modelo hasta ahora desarrollado.

En febrero del 2018, Ackman anunció su desistimiento con la apuesta realizada contra Herbalife después de cinco años en los cuales la compañía fue ampliamente cuestionada y auditada.

No obstante, es importante dilucidar ciertos aspectos importantes.

- En principio, una empresa multinivel posee una estructura de distribuidores y vendedores, siendo que los ingresos obtenidos son de acuerdo a los productos vendidos propios y de aquellos quienes trabajan bajo su supervisión.

- El ofrecimiento no solo consta de productos, sino también de servicios y, puede desarrollarse en ámbitos de salud, maquillaje, etc. Un gran ejemplo son las empresas Avon y Esika.

- Una empresa multinivel no es ilegal por sí misma, en tanto los ingresos provengan mayormente de los productos vendidos.

De acuerdo al documental mencionado, Peter VanderNat, Economista Senior de la Comisión Federal de Comercio, determina que "Una organización es un esquema piramidal cuando los participantes obtienen más beneficios monetarios de reclutar que de vender productos y servicios a los consumidores." Lo cual significó un fuerte argumento para ir en contra de Herbalife.

Es importante que una empresa multinivel aloje un ambiente ordenado $y$, tratándose de un negocio en el que priman las prestaciones recíprocas, es necesaria una investigación más a fondo a cargo de la Superintendencia.

Tomando en cuenta el punto de críticas en el que los productos de Herbalife suelen ser demasiado costosos, Carlos Cueva, el jefe del Departamento de Asuntos Contenciosos de la SBS, declaró que se trata de decisiones netamente tomadas por los consumidores, puesto que son ellos quienes determinan si compran o no o, si quieren ser distribuidores o no.

En este plano, es importante tener claro que cuando se trata de una empresa multinivel, se está hablando de constantes contratos de compra-venta, con estipulaciones marcadas y, no de empresas basadas en reclutar personas y captar dinero, siendo esta una diferencia bastante marcada. 
Sin embargo, ello no implica que una empresa multinivel no puede ser usada de fachada para la realización de actos ilícitos como el de albergar un esquema Ponzi, sino por el contrario, puede ser la mejor de las ideas desde el punto de vista de una mente criminal.

Otro punto importante de diferencia entre ambas consiste en la 'captación de personas', puesto que para ingresar a ser distribuidora de una multinivel no es necesario o requisito exigible el llevar a dos personas más, de hecho, a pesar de ser una sugerencia por los supervisores, no pasa de ello. Sin embargo, debido a que se trata de un esquema piramidal y su actividad se basa en el incremento del grupo de personas, muchas veces la "sugerencia" pasa por exigencia.

Asimismo, el gremio que reúne a muchas empresas multinivel, entre ellas las grandes empresas de venta de productos por catálogo como Belcorp, la Cámara Peruana de Ventas Directas (Capevedi), expresa que una diferencia importante es el monto de ingreso al sistema. En tanto a empresas multinivel, el costo no es demasiado alto y es retribuible con productos de campaña. Pero al tratarse de esquemas Ponzi, estos buscan poseen tarifas demasiado altas.

\section{Recomendaciones de la SBS en Perú, después del caso CLAE.}

A partir del caso CLAE, la SBS ha ido fortaleciendo sus programas de proceder y combatir la informalidad financiera, para cumplir con preservar la economía y salvaguardar el ahorro del público, a fin de evitar con ello crisis sociales y económicas tan grandes como esas.

Por ejemplo, el Perú fue representado en el Primer Seminario Internacional Sobre Estafas Piramidales realizada en el 2008 en Bolivia, donde se expusieron los concerniente a esta modalidad de estafa y, entre otros temas, se tomó en consideración de exposición el caso CLAE y, las medidas que está tomando la SBS para prevenir entidades informales. Dentro de estas medidas se encuentran: "Potenciar las acciones de detección de la UIF insertada a la SBS desde 2007. Cuenta con personal calificado y se especializa en la investigación de lavado de activos..." Así como "fomentar el desarrollo de microfinanzas, convertirlas de informales a formales" y difundir los riesgos en los que incurre el público si forma parte de un sistema piramidal".

Prueba de ello se encuentran en las distintas publicaciones que realiza la entidad para informar sobre el tema y no ser víctimas de estafas con esas. A consideración de ello, la SBS emitió algunos "tips" al público en general, de entre los que destacan:

- Verifica siempre la identidad, domicilio y existencia formal de las personas o empresas con las que haces negocios, y documenta toda transacción.

- Desconfía siempre del ofrecimiento de altas tasas de interés o de altas ganancias en cortos periodos de tiempo. Para ello, compara dicho ofrecimiento con la ganancia que se obtiene en el mercado financiero formal.

Asimismo, la Superintendencia ofrece consultas o la posibilidad de que el público realice denuncias en caso se tenga sospechas de la existencia de una pirámide financiera, vía telefónica o correo electrónico.

\section{Análisis de la cuestión}

Tomando en cuenta lo ya desarrollado a lo largo de la investigación, es muy fácil plantearse una posición en el caso CLAE, a partir de ello, los esquemas piramidales han sido de frecuente aparición en el sistema financiero peruano. 
Muchos de los denominados claeístas aún siguen exigiendo la devolución de su dinero, ahora con 20 años más encima. Algunos otros dejaron de persistir en el proceso y, a otros "afortunados" se les ha hecho devolución de un porcentaje mínimo de lo aportado.

Sin embargo, el punto central se encuentra en los testimonios de personas que fueron víctimas del esquema CLAE pero que, aun así, sabiendo que se encontraban frente a otra igual siguieron en pie con la idea de aportar y arriesgarse al todo o nada, perdiendo de esa manera, por vez repetida, los ahorros juntados.

El caso que traigo a colación es el referido a la empresa "Broker Market Internacional S.A.C.", el cual fue intervenido en el año 2008 y, a pesar de no consistir en una pirámide a gran escala como CLAE, logró persuadir de aportar a ex claeístas.

En el Noticiero Panorama del año 2009 tres testimonios de personas que fueron víctimas de CLAE y, que sabían que consistía en una pirámide afirman perderlo todo por arriesgarlo en esta empresa, pues le ofrecían no pagar impuestos. Sus testimonios datan desde, "no sabía que duraría tan poco" y "fue mi hijo de 10 años quien me advirtió".

"En ese sentido, cuando se trata de confianza en el sistema financiero, se dice que, "la confianza del cliente en su banco o caja de ahorro es (...) absoluta (...) estas tienen por actividad típica y habitual recibir fondos del público". (LÓPEZ, 2009. p.3)

Estas personas realizaron esas operaciones aun sabiendo que podían perderlo todo. Siendo de esta manera y, teniendo en cuenta las medidas que toma la SBS, en muchos de los casos no podría tratarse de falta de información.

\section{Conclusiones}

Las medidas de la SBS a pesar de llegar a ser acertadas, resultan ser insuficientes para que las personas desistan de aportar su dinero en esquemas piramidales aun sabiendo que se trata de un esquema fraudulento. A pesar que se dice que es la posible falta de información, esto no siempre sucede así y lo que determina si una persona confía en una determinada entidad financiera no es plenamente la confianza, sino la falta de educación financiera que impida el incurrir en esto. En tal sentido, los objetivos propuestos por la autoridad parecen permanecer aún en la bandeja de pendientes.

Sin embargo, es posible que sea, en esta oportunidad, la propia autoridad quien deba hacer una revisión juiciosa y profunda de sus compromisos, con el objeto de ponderar si en los tiempos actuales, el actuar único y aislado el estado, es suficiente para generar impactos intergeneracionales y de alcance expansivo que se busca, en la sociedad en su conjunto. La experiencia comparada ha demostrado que los verdaderos cambios culturales y sociales se manifiestan cuando el estado involucra y establece alianzas con el mundo privado, con el fin de alcanzar los objetivos sociales que se ha propuesto a la luz de los principios constitucionales e internacionales vinculados al Desarrollo y los Derechos Humanos. ¿Los cambios no se producirían de forma más precipitada si todo el sector privado financiero trabajara, de forma comprometida y profunda, en transparentar el contenido y forma de funcionamiento del mercado y de los diferentes instrumentos financieros, entregando Educación Financiera de calidad a todos los peruanos que tenga a su alcance? ¿Esa no sería una forma efectiva y directa de impedir la migración de ciudadanos peruanos al sistema informal, a través de la amplia difusión de las ventajas y garantías que a largo plazo presenta el Sistema Financiero y Bancario formal? 
Tales preguntas no resultan descabelladas, desde el momento en que las estafas piramidales (al menos aquellas de la magnitud de las relatadas en este breve texto) han perjudicado a todo el sistema financiero en su conjunto, causando daños cuantitativos y cualitativos imponderables. No se podría concluir otra cosa después de examinar cada uno de los casos y el desarrollo reiterado de estos fenómenos, en diferentes épocas y países.

¿Sería diferente el escenario si los Bancos del Perú comenzaran a plantearse profundamente los riesgos reales que suponen para ellos la precarización del sistema financiero del Perú al largo plazo y, dentro de sus atribuciones, propusieran medidas innovadoras para encantar a los peruanos y despejar el halo de duda y escepticismo que existe sobre ellos? Parece algo urgente en lo que trabajar y una materia no sólo de interés estatal.

\section{Referencias:}

- ACOSTA, G. (2018) Temas sobre Derecho de Protección al Consumidor y Regulación financiera - Círculo Derecho Administrativo. Lima: Tarea Gráfica Editores, 2018

- Autoridad de Supervisión del Sistema Financiero. (2009) Estafas Piramidales: Lecciones aprendidas. ASFI. Bolivia. [en línea]. Recuperado de: https://www.asfi.gob.bo/images/ASFI/ DOCS/LIBROS/EstafasPiramidales.pdf

- CONGER, INGA Y WEBB. (2015). El árbol de la mostaza: Historias de las Microfinanzas en el Perú USMP. Lima, Perú. [en línea]. Recuperado de: http://usmp.edu.pe/idp/wp- content/ uploads/2015/08/el_arbol_mostaza_microfinanzas_web.pdf

- EL COMERCIO. (2017). CLAE: Historia y testimonio de la estafa más grande del país. Lima, Perú. [en línea]. Recuperado de: https://elcomercio.pe/economia/peru/clae-historiatestimonios-estafa-grande-pais-231916

- EL COMERCIO. (2017). Empresario es acusado de estafar al mismo estilo de CLAE. Lima, Perú. [en línea]. Recuperado de: https://elcomercio.pe/lima/judiciales/empresario-acusadoestafar-estilo-clae-noticia-478781

- FORBES. (2018). Bill Ackman Ends 5-Year Battle Against Herbalife. (en línea). Recuperado de: $\quad$ https://www.forbes.com/sites/gurufocus/2018/02/28/bill-ackman-ends-5-year-battleagainst-herbalife/\#2fc6ab619838

- GALLEGO, J \&NAVAS, J. (2008). ¿Còmo pudo Madoff estafar a ricos y grandes de la banca? El mundo.es. (en línea). Recuperado de: https://www.elmundo.es/mundodinero/2008/12/16/ economia/1229435860.html

- REDACCIÓN GESTIÓN. (2017). SBS: ¿Son consideradas estafas piramidales las ventas multinivel? Gestión. (en línea). Recuperado de: https://gestion.pe/tu-dinero/sbs-sonconsideradas-estafas-piramidales-ventas-multinivel-141182

- LA REPÚBLICA (2009). Veinte personas fueron detenidas por estafas "pirámide". [en línea]. Recuperado de: https://larepublica.pe/sociedad/387450-veinte-personas-fueron-detenidaspor-estafas-piramide

- LÒPEZ, J. (2009). El sistema financiero: una cuestión de confianza. UNED. La Ley, grupo Wolter Kluwer. Malaga, España. [en línea]. Recuperado de: https://www2.uned.es/expertodinero-banca/trabajos/El_Sistema_Financiero Una_cuestion_de_confianza.pdf

- MARTELL, S. (2017). ¿Qué es CLAE y quién es Carlos Manrique Carreño? El Comercio. Lima, Perú. (en línea). Recuperado de: http://rpp.pe/economia/economia/que-es-clae-yquien-es-carlos-manrique-carreno-noticia-1022538

- REDACCIÓN GESTIÓN. (2016). Estafa piramidal: ¿Cómo diferenciarla de una red legal de ventas multinivel? (en línea). Recuperado de: https://gestion.pe/tu-dinero/estafa-piramidaldiferenciarla-red-legal-ventas-multinivel-147186 
- REDACCIÓN GESTIÓN. (2017). Fondo para víctimas de Bernard Madoff haría primer pago este año. Gestión. (en línea). Recuperado de: https://gestion.pe/economia/mercados/fondovictimas-bernard-madoff-haria-primer-pago-ano-137509

- REDACCIÓN GESTIÓN. (2016). SBS investiga estafa piramidal que afecta a varias familias limeñas adineradas. Gestión. (en línea). Recuperado de: https://gestion.pe/tu-dinero/sbsinvestiga-estafa-piramidal-afecta-familias-limenas-adineradas-146763

- REDACCIÓN RPP. (2016). ¿Còmo operan los telares de sueños? La nueva estafa piramidal. RPP. (en línea). Recuperado de: https://rpp.pe/economia/economia/como-operan- telaresde-suenos-nueva-estafa-piramidal-noticia-977660

- RPP. (2016). Video: alertan sobre 'Tejedoras de sueños', la nueva estafa pirámide en Perú. Lima, Perú. [en línea]. Recuperado de: http://rpp.pe/economia/economia/tejedoras-desuenos-alertan-sobre-nueva-estafa-piramide-noticia-977373

- PAREJO, I. (2017). La estafa piramidal: Un estudio exploratorio de la víctima. Journal of Negative\&no Positive Reults. Universidad de Sevilla, España. (en línea). Recuperado de: (http://revistas. proeditio.com/jonnpr/article/view/1237/pdf1237)

- SUPERINTENDENCIA DE BANCA, SEGUROS Y AFP. (2017). Las "pirámides financieras" y la certeza de perder nuestros ahorros. SBS Informa, boletín quincenal No11. Lima, Perú. [en línea]. Recuperado de: http://www.sbs.gob.pe/Portals/0/jer/BOL- QUINCENAL/20170714_ BolQuincenal-N11.pdf

- SMV. (2018). Finalidad y Funciones. (en línea). Recuperado de: http://www.smv.gob.pe/ Frm_VerArticulo?data=17B15B848FCE8F37FA86E13166C6752043 C6DCB32142B82 3F43909D41274C8008858C8• Codigo Penal Peruano http://spij.minjus.gob.pe/content/ publicaciones_oficiales/img/CODIGOPENAL.pdf

- Betting on Zero, Netflix. Video•Panorama. (2009). BMI=CLAE parte1. (en línea). Recuperado de: https://www.youtube.com/watch?v=O-ztkbweyO0

Fecha de recepción: 23 de octubre de 2020

Fecha de aceptación:06 de noviembre de 2020 